\title{
Esophageal metastases of hepatocellular carcinoma following liver transplantation
}

\author{
Bruno Škurla, Aleksandra Mlinarić, Sergej Nadalin \\ Miroslava Katičić, Slavica Naumovski-Mihalić
}

\begin{abstract}
Introduction: Distal metastases occur in 30 to 75\% cases of advanced hepatocellular carcinoma and the most common sites are the lungs, bones, intraperitoneal organs and adrenal gland. The most frequent metastases, except those mentioned above, are in the regional lymph nodes. Metastases to the esophagus are very rare, being present in less than $0.4 \%$ of patients with hepatocellular carcinoma. Case Report: We present a case of a 56-year-old male patient who had been treated for alcoholic liver cirrhosis and hepatocellular carcinoma in cirrhotic liver, followed by orthotopic liver transplantation (OLT). Three months after OLT patient was admitted to the hospital due to anemia. The upper gastrointestinal endoscopy revealed two lesions in the lower part of the esophagus, of which the lesion at the cardia severely bleed. Sclerotherapy was the method of choice in order to stop bleeding which was followed by surgery after sclerotherapy failed. Biopsies of the esophageal lesions showed metastases of hepatocellular carcinoma, while multiple metastases in the lungs and the mediastinum
\end{abstract}

Bruno Škurla ${ }^{1}$, Aleksandra Mlinarić ${ }^{1}$, Sergej Nadalin ${ }^{2}$, Miroslava Katičić ${ }^{1}$, Mihalić Slavica Naumovski ${ }^{1}$ Affiliations: ${ }^{1}$ Department of gastroenterology, Clinical hospital, Merkur, Zagreb, Croatia; ${ }^{2}$ Department for Biology and Medical Genetics, School of Medicine, University of Rijeka, Croatia.

Corresponding Author: Aleksandra Mlinaric, MD, Clinical

Hospital, Merkur, I. Zajca 19, 10000 Zagreb, Croatia;

Phone/Fax: +38512431393;

Email: aleksandramlinaric@yahoo.com

Received: 26 September 2010

Accepted: 4 November 2010

Published: 19 December 2010 had been diagnosed earlier. After the patient had recovered from surgery sorafenib was suggested as adjuvant therapy. The patient gave up on further treatment and eventually passed away. Conclusion: The presently available scanning methods in severely ill patients will not always detect extrahepatic metastases of the liver cancer, thus causing an inappropriate use of liver transplantation. OLT failure resulting in spreading of the hepatocellular carcinoma will make us redefine the criteria for liver transplantation.

Keywords: Malignant diseases of the liver, Alcoholic liver cirrhosis, Hepatocellular carcinoma, Orthotopic liver transplantation, Esophageal metastases

$$
* * * * * * * * *
$$

Škurla B, Mlinarić A, Nadalin S, Katičić M, Naumovski MS. Esophageal metastases of hepatocellular carcinoma following liver transplantation. International Journal of Case Reports and Images 2010;1(4):7-11.

$$
* * * * * * * * * \text {. }
$$

doi:10.5348/ijcri-2010-12-10-CR-2

\section{INTRODUCTION}

Primary malignant diseases of the liver can be treated by orthotopic liver transplantation (OLT) if the extent of the disease is not too great. Unfortunately, it is difficult to find small and microsopic metastases which allow fast spreading in an immunosuppressed patient. Distal metastases, excluding regional lymph nodes, occur in 30 to $75 \%$ cases of advanced 
hepatocellular carcinoma [1] and the most common sites are the lungs, bones, intraperitoneal organs and adrenal gland [2-5]. Metastases to the esophagus are very rare being present in less than $0.4 \%$ of patients with hepatocellular carcinoma (HCC) $[6,7]$.

We report the first case of multiple, intermitently bleeding metastases of hepatocellular carcinoma in the esophagus in Europe, diagnosed three months after liver transplantation. Only two cases of unusual spreading of $\mathrm{HCC}$ in the oesophagus have been reported so far both in China $[1,8]$.

\section{CASE REPORT}

A 56-year-old male Caucasian, who had been treated for five years due to alcoholic cirrhosis, was admitted to hospital in October, 2008, because of single hematemesis. Endoscopy revealed esophageal varices (Figure 1), without actual bleeding. Sclerotherapy was performed, packed cells transfusion ordered, and there was no re-bleeding during hospitalization. Ultrasound and computed tomography (CT) scan revealed three liver lesions, ranging in size from $2.0 \mathrm{~cm}$ to $3.3 \mathrm{~cm}$. Alpha-fetoprotein, viral serology markers (hepatitis A, B and C), colonoscopy and chest X-ray findings were normal. Abdominal magnetic resonance (MR) scan was indecisive, but suggested a possibility of liver cancer of the left lobe (segment 4) and right lobe (segment 7 and 8) of the liver. MR imaging procedures (fast imaging employing steady state acquistion-FIESTA, fast relaxation fast spin echo-FRFSE T2, fast spin echo, spin spin relaxation time- FSE T2, DUAL ECHO, fast spoiled gradient echo spin lattice relaxation time-FSPGR T1) were performed with paramagnetic contrast in all scans. Due to ascites and coagulopathy histology or cytology of the described lesions were not possible. Neither of the imaging techiques showed signs of portal vein thrombosis. The patient was discharged so

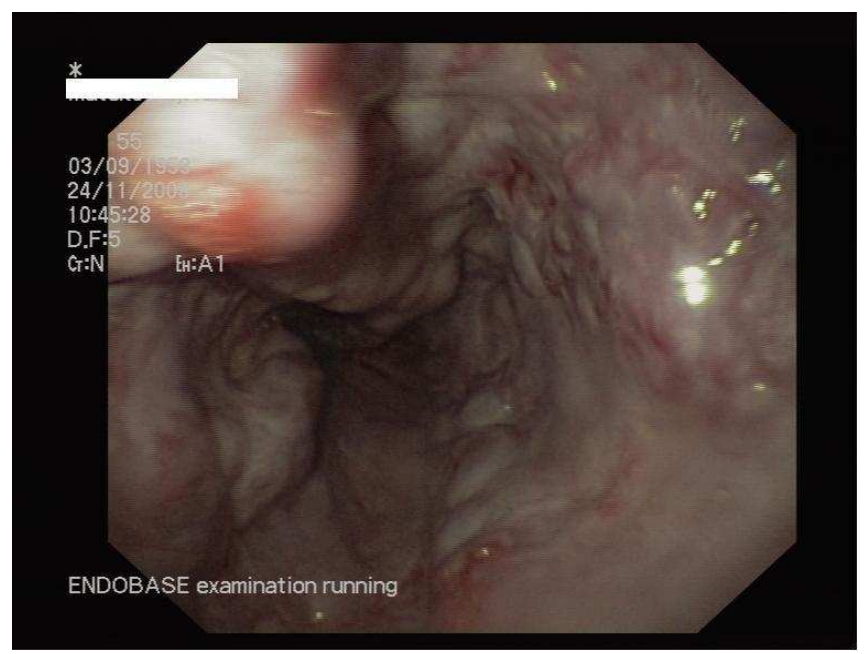

Figure 1: Esophageal varices. we could assess esophageal varices later, but within a month the patient was readmitted again due to upper gastrointestinal (GI) bleeding. Again, no actual site of bleeding was found, so we performed endoscopic band ligation on the varices.

After the patient was stabilized, needle aspiration of the liver lesions was done which established the diagnosis of HCC. Again, the presence of ascites and coagulopathy made fine needle aspiration (cytology aspiration) more appropriate in obtaining the diagnosis instead of biopsy. The smear showed atypical cells resembling hepatocytes, with marked anysocytosis and anisonucleosis. The cells were CK18 positive and, CK19 and CK2O negative. The cytology diagnosis was 'hepatocellular carcinoma'. Other than usual exhaustion from bleeding and parenteral feeding the patient had no other leading symptoms like dysphagia, odynophagia or substantial weight loss. The patient was, after standard procedure that included upper and lower GI endoscopy, establishing viral status of the patient, excluding the hepatorenal and hepatopulmonary syndroma, otorhinolaryngeal and urology examination with complete biochemistry, reported for The National Liver Transplantation list with Model for End Stage Liver Disease (MELD) score 16, and received a new organ in January, 2009. The surgery went well; the explanted liver was examined by an experienced pathologist. Serial slices of the left liver lobe showed multiple tumors ranging in size from 0.2 $\mathrm{cm}$ to $2.0 \mathrm{~cm}$, spreading to the right lobe of the liver where five tumor masses, ranging from $0.5 \mathrm{~cm}$ to 2.5 $\mathrm{cm}$ were found.

The portal vein was invaded and occluded by cancer cells, as well as the blood vessels in the ligament of teres. The surrounding tissue next to the liver was also invaded by the tumor. The preserved part of the liver parenchyma showed signs of an incomplete cirrhosis, even though its blood vessels showed signs of tumor invasion. The histology finding matched completely with previous cytology findings.

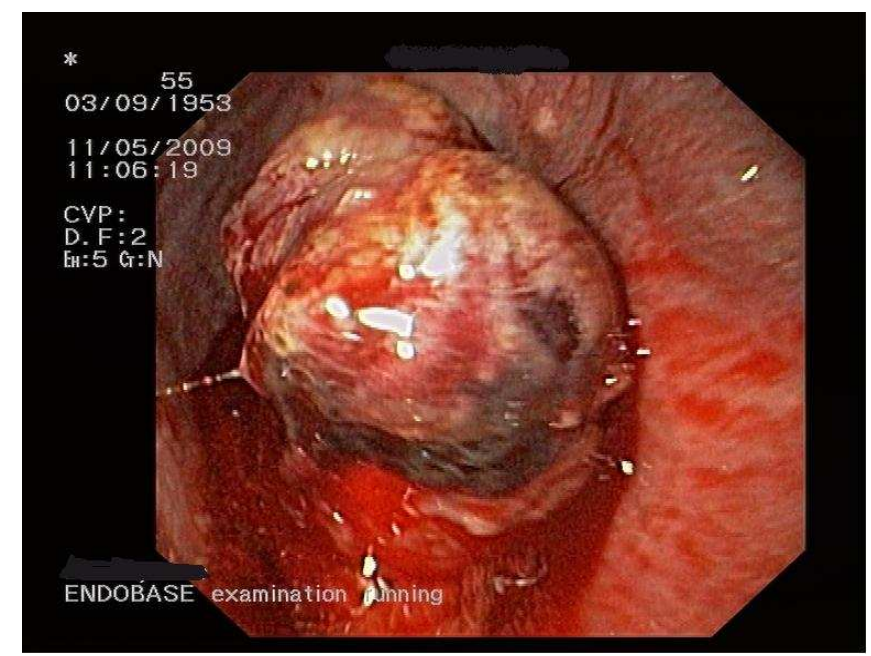

Figure 2: Bleeding mass expanding from cardia. 
At the beginning of May 2009, the patient was admitted to the hospital again due to anemia. Gastroscopy revealed a lesion (Figure 2) at the end of the esophagus, which was intermittently spurting blood. At first sight, it was unclear if it was a polyp, large blood clot, or a varix. Five days later the lesion prolapsed into the fundic part of the stomach with venous bleeding (Figure 3) which was stopped by sclerotherapy, even though the tissue was unusually soft and yielding to the catheter. Five days later we noticed a flat lesion (Figure 4), five $\mathrm{cm}$ above the cardia, elevated from the surrounding mucosa, $1.5 \mathrm{~cm}$ in diameter. Even though the lesions bled intermittently, biopsies were taken which revealed a HCC metastase in both lesions. The chest X-ray revealed multiple lesions within the lungs (Figure 5). Brain and abdominal CT scan revealed no other lesions. Since the patient bled from the esophageal lesion every time he underwent upper GI endoscopy, there was no satisfactory mean to solve the problem via endoscopy, so surgery was chosen in order to stop bleeding. Intraoperatively, the cardiac metastasis significantly bled, so it was resected and sutured. At laparotomy it was clear that the transplanted liver and surrounding lymph nodes were packed with metastases. Multiple biopsies were obtained from the metastasis. It was clear there was no surgical solution for the extensive stage of the metastatic disease. The oesophageal resection was merely a life saving procedure. The patient came for regular check-ups in his surgery recovery period.

His last visit was in July, 2009 and he was stable. Irradiation as a therapeutic option was discarded because of the expected complications in massively spread malignant disease. Sorafenib adjuvant therapy was suggested, but the patient developed a serious case of depression, and lost the will to carry on. Eventually the patient passed away, but the information on that is indirect and off the record.

\section{DISCUSSION}

During the last ten years only a few cases of hepatocellular carcinoma with esophageal metastases have been reported $[1,6,8-12]$. Two articles on esophageal metastases of hepatocellular carcinoma followed by OLT have been published so far $[1,8]$. The leading symptom in our case as well as in case described by Hsu et. al. [8] was bleeding from the upper GI tract, while in case described by Xie et. al. [1] dysphagia and odynophagia were leading symptoms. Biopsies of lesions found at endoscopy brought up exact diagnosis in all cases described above 'metastases of hepatocellular carcinoma'. Hsu et. al. applied conservative therapy in order to stop bleeding, but the disease progressed and the patient died within a couple of months [8]. In our case, massive bleeding in the esophagus required life saving surgery.

Recovering from surgery, sorafenib was suggested

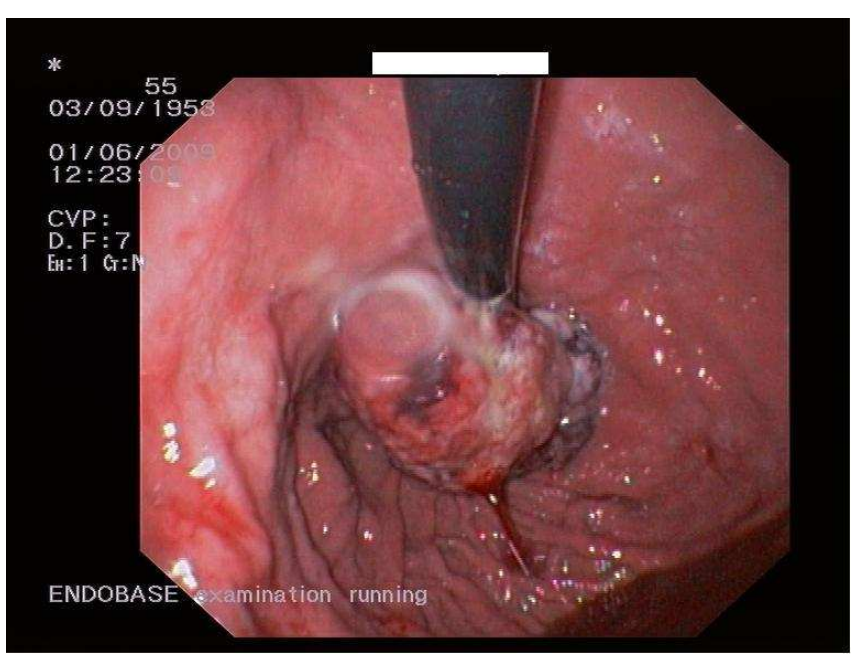

Figure 3: Actual bleeding of the metastase.

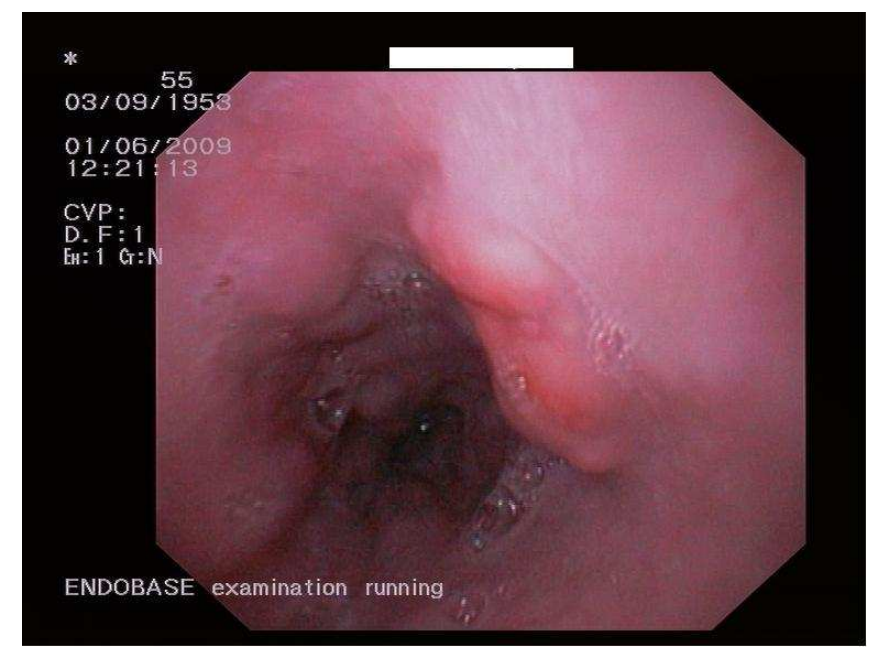

Figure 4: Non bleeding metastase in the esophagus.

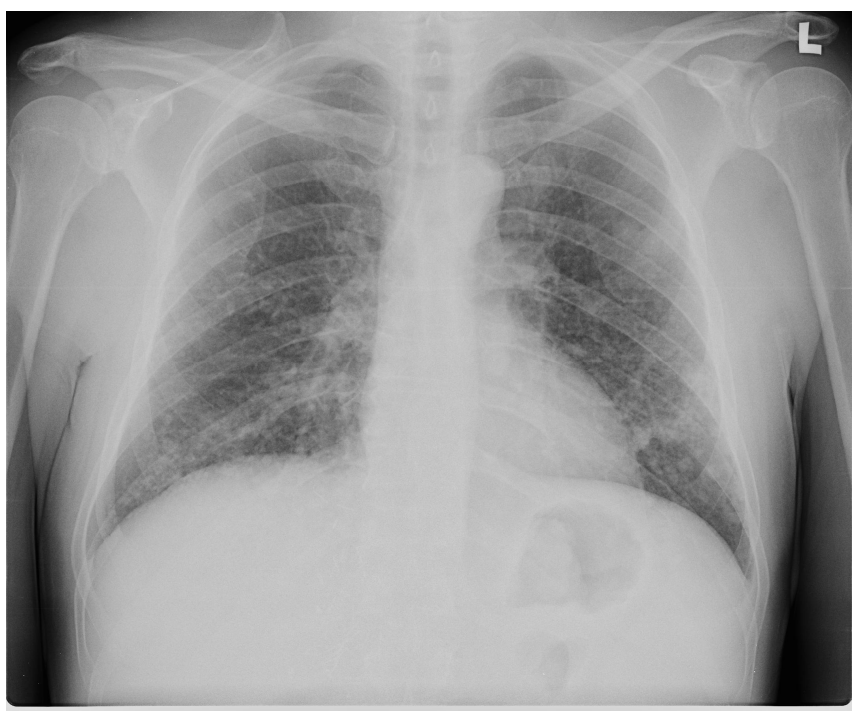

Figure 5: Chest X-ray revealed multiple lesions within the lungs. 
as adjuvant therapy and the patient was stable at following check-ups for six months after OLT, till July 2009. Later on, the patient developed a serious case of depression and lost the will to carry on, passing away eventually. Xie et. al. found metastases of hepatocellular carcinoma solely in the esophagus which were treated with radiotherapy [1]. After more than six months of radiotherapy the endoscopy could not detect metastatic lesions in the esophagus, while the lesion in the liver was stable. It is presumed that tumor invasion of portal veins caused tumor infested thrombi which are disseminated throughout GI system [12, 13]. Patients receiving immunosuppressive therapy are supposed to be more prone to develop metastases of earlier diagnosed malignant diseases. They also develop primary malignant diseases other than hepatic cancer more frequently [1].

Due to their dimensions, dull clinical course and short interval between sowing in of esophageal metastases and death, the most of patients are not diagnosed until post mortem [13]. Orthotopic liver transplantation is the method of choice in well chosen patients with terminal liver diseases [14]. In our Institution the Milan criteria and MELD score [15] are the usual inclusion methods for liver transplantation. Available morphological examinations and scanning do not always provide satisfactory staging and the extent of the disease. Macronodular cirrhosis can mimic hepatocellular carcinoma and vice versa. Concomitant disorders like ascites and coagulopathy makes establishing the diagnosis much more complicated. In this case, all imaging studies didn't match the actual spread of the extrahepatic metastases. Unfortunately, the explanted liver showed poor prognostic features in our case - multiple tumors in both lobes of the liver, vascular invasion and the tumor invasion of the surrounding tissue. It is impossible to pinpoint the risks responsible of the esophageal spread of the disease. Small number of similar cases reported so far confirms this statement. Our assumption that the immunosupressive therapy may contribute to the spread of the metastases is yet to be confirmed in well designated studies, and is not the object of this case report. Metastases of hepatocellular carcinoma including metastases in the esophagus can be treated with surgery, radiotherapy and sorafenib, thus extending the life expectancy. Sorafenib inhibits tumor cell proliferation and tumor angiogenesis [16]. Used as palliative chemotherapy it can be delivered to patients with recurrent hepatocellular carcinoma after liver transplantation with tolerable toxicity, but the efficacy to date is not satisfactory [17]. However, in patients with advanced hepatocellular carcinoma, median survival and the time to radiologic progression were nearly three months longer for patients treated with sorafenib than for those given placebo [18]. In order to make the diagnosis of metastatic spreading of hepatocellular carcinoma in the esophagus, the upper GI endoscopy is essential in both visualizing and sampling the lesion. If the latter is unsatisfactory other methods can be useful such as CT scan or angiography.
Unfortunately, the presently available scanning methods in severely ill patients will not always reveal extrahepatic metastases of the liver cancer, thus causing an inappropriate use of liver transplantation. Our efforts will have to be emphasized on finding distant metastases after establishing the diagnosis of the hepatocellular carcinoma, thus choosing the right patient eligible for liver transplantation. It is not easy to decide to expose all patients to invasive procedures like laparoscopy, repetitive bronchoscopies, upper GI endoscopies etc., but the OLT failure resulting in spreading of the hepatocellular carcinoma will make us redefine the criteria for liver transplantation.

\section{CONCLUSION}

Unfortunately, the presently available scanning methods in severely ill patients will not always reveal extrahepatic metastases of the liver cancer, thus causing an inappropriate use of liver transplantation. Our efforts will have to be emphasized on finding distant metastases after establishing the diagnosis of the hepatocellular carcinoma, thus choosing the right patient eligible for liver transplantation. It is not easy to decide to expose all patients to invasive procedures like laparoscopy, repetitive bronchoscopies, upper GI endoscopies etc., but the OLT failure resulting in spreading of the hepatocellular carcinoma will make us redefine the criteria for liver transplantation.

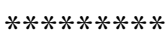

\section{Author Contributions}

Bruno Škurla - Conception and design, Acquisition of data, Analysis and interpretation of data, Drafting the article, Critical revision of the article, Final approval of the version to be published

Sergej Nadalin - Conception and design, Acquisition of data, Analysis and interpretation of data, Final approval of the version to be published

Aleksandra Mlinarić - Conception and design, Acquisition of data, Analysis and interpretation of data Miroslava Katičić - Drafting the article, Critical revision of the article, Final approval of the version to be published

Mihalić Slavica Naumovski - Drafting the article, Critical revision of the article

\section{Guarantor}

The corresponding author is the guarantor of submission.

\section{Conflict of Interest}

Authors declare no conflict of interest.

\section{Copyright}

(C) Bruno Škurla et. al. 2010; This article is distributed under the terms of Creative Commons attribution 3.0 
License which permits unrestricted use, distribution and reproduction in any means provided the original authors and original publisher are properly credited. (Please see www.ijcasereportsandimages.com /copyright-policy.php for more information.)

\section{REFERENCES}

1. Xie LY, Fan M, Fan J, Wang J, Xu XL, Jiang GL. Metastatic hepatocellular carcinoma in the esophagus following liver transplantation. Liver Transpl 2008;14: 1680-1682.

2. Ho J, Wu PC, Kung TM. An autopsy study of hepatocellular carcinoma in Hong Kong Pathology 1981; 13: 409-416.

3. Nakashima T, Okuda K, Kojiro M, Jimi A, Yamaguchi R, Sakamoto K et. al. Pathology of hepatocellular carcinoma in Japan. 232 Consecutive cases autopsied in ten years. Cancer 1983;51: 863877.

4. Yuki K, Hirohashi S, Sakamoto M, Kanai T, Shimosato Y . Growth and spread of hepatocellular carcinoma. A review of 240 consecutive autopsy cases. Cancer 1990;66: 2174-2179.

5. Kaczynski J, Hansson G, Wallerstedt S. Metastases in cases with hepatocellular carcinoma in relation to clinicopathologic features of the tumor. An autopsy study from a low endemic area. Acta Oncol 1995;34: 43-48.

6. Tsubouchi E, Hirasaki S, Kataoka J, Hidaka S, Kajiwara T, Yamauchi Y et al. Unusual metastasis of hepatocellular carcinoma to the esophagus. Intern Med 2005;44: 444-447.

7. No authors listed The Liver cancer study group of Japan. Primary liver cancer in Japan Annals of Surgery 1990;211: 277-287.

8. Hsu KF, Hsieh TY, Yeh CL, Shih ML, Hsieh CB. Polypoid esophageal and gastric metastases of recurrent hepatocellular carcinoma after liver transplantation. Endoscopy 2009;41: E82-E83.

9. Sohara N, Takagi H, Yamada T, Ichikawa T, Abe T, Itoh H. Esophageal metastasis of hepatocellular carcinoma. Gastrointest Endosc 2000;51: 739-741.

10. Kume K, Murata I, Yoshikawa I, Kanagawa K, Otsuki M. Polypoid metastatic hepatocellular carcinoma of the esophagus occurring after endoscopic variceal band ligation. Endoscopy 2000;32: 419-421.

11. Cho A, Ryu M, Yoshinaga Y, Ishikawa Y, Miyazawa Y, Okazumi S et. al. Hepatocellular carcinoma with unusual metastasis to the esophagus. Hepatogastroenterology 2003;50: 1143-1145.

12. Arakawa M, Kage M, Matsumoto S, Akagi Y, Noda $\mathrm{T}$, Fukuda K et. al. Frequency and significance of tumor thrombi in esophageal varices in hepatocellular carcinoma associated with cirrhosis. Hepatology 1986;6:419-422.

13. Choi CS, Kim HC, Kim TH, Seo GS, Kim KH, Cho EY et. al. Does the endoscopic finding of esophageal metastatic hepatocellular carcinoma progress from submucosal mass to polypoid shape? Gastrointest Endosc 2008;68: 155-159.

14. Arii S, Monden K, Niwano M, Furutani M, Mori A, Mizumoto M et. al. Results of surgical treatment for recurrent hepatocellular carcinoma; comparison of outcome among patients with multicentric carcinogenesis, intrahepatic metastasis, and extrahepatic recurrence. J Hepatobiliary Pancreat Surg 1998;5: 86-92.

15. Jonas S, Bechstein WO, Steinmüller T, Herrmann $\mathrm{M}$, Radke C, Berg T et. al. Vascular invasion and histopathologic grading determine outcome after liver transplantation for hepatocellular carcinoma in cirrhosis. Hepatology 2001;33: 1080-1086.

16. Wilhelm SM, Carter C, Tang L, Wilkie D, McNabola A, Rong $\mathrm{H}$ et. al. BAY 43-9006 exhibits broad spectrum oral antitumor activity and targets the $\mathrm{RAF} / \mathrm{MEK} / \mathrm{ERK}$ pathway and receptor tyrosine kinases involved in tumor progression and angiogenesis. Cancer Res 2004;64:7099-7109.

17. Lee JO, Kim DY, Lim JH, Seo MD, Yi HG, Oh DY et. al. Palliative chemotherapy for patients with recurrent hepatocellular carcinoma after liver transplantation Journal of gastroenterology and hepatology 2009;24:800-805.

18. Llovet JM, Ricci S, Mazzafero V, Hilgard P, Gane E, Blanc JF et. al. Sorafenib in advanced hepatocellular carcinoma The New England Journal of Medicine 2008;359:378-390. 